\title{
Adsorption of random copolymers: A scaling analysis
}

K. Sumithra, and A. Baumgaertner

Citation: The Journal of Chemical Physics 110, 2727 (1999); doi: 10.1063/1.477996

View online: https://doi.org/10.1063/1.477996

View Table of Contents: http://aip.scitation.org/toc/jcp/110/5

Published by the American Institute of Physics

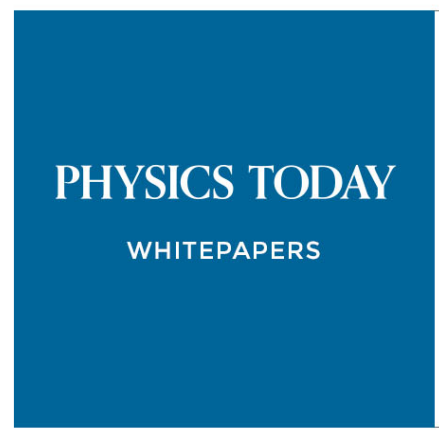




\title{
Adsorption of random copolymers: A scaling analysis
}

\author{
K. Sumithra and A. Baumgaertner ${ }^{\mathrm{a})}$ \\ Forum Modellierung, Forschungszentrum, 52425 Jülich, Germany
}

(Received 10 August 1998; accepted 29 October 1998)

\begin{abstract}
We report on results from Monte Carlo simulations of a single random copolymer adsorbed on a homogeneous planar surface. Although the critical crossover exponent is unaltered with respect to the case of homogeneous polymers, it is found that the scaling behavior is changed by the fraction of adsorptive monomers of the chain. In particular, we present some explicit expressions for energy and radius of gyration at low temperatures. (C) 1999 American Institute of Physics.
\end{abstract}

[S0021-9606(99)51305-6]

\section{INTRODUCTION}

Owing to the enormous practical interest, ranging from biological physics to biosensor and pattern recognition applications, polymer adsorption on surfaces has been the focus of a broad spectrum of experimental and theoretical investigations. In particular, since many polymers possess heterogeneous compositions (e.g., proteins) there is much interest in determining the relation between their compositions and their adsorption characteristics.

Beside polymers with specific sequences (e.g., proteins) there has been considerable interest in random copolymers serving as a paradigmatic model for more realistic heterogeneous macromolecules. Among others, studies have been performed on the localization of random copolymers at interfaces, ${ }^{1-4}$ the adsorption of random polyampholytes, ${ }^{5,6}$ the adsorption-freezing transition of random copolymers near disordered surfaces, ${ }^{7,8}$ and the effect of specific sequence distributions on the adsorption transition. ${ }^{9,10}$ In a series of papers Gutman and Chakraborty ${ }^{11,12}$ have developed a replica-based mean-field theory for random block copolymers interacting with chemically disordered surfaces.

In the present paper we address the questions concerning the critical and low temperature properties of random copolymers at a homogeneous adsorbing surface. In particular, we have examined the question of how much random copolymers differ from homopolymers ${ }^{13,14}$ with respect to their critical behavior.

The study of adsorption of random copolymers on surfaces merits attention because the physical status of the problem is somewhat different from the pure polymer adsorption. In the latter case, each monomer has active attractive interactions with the adsorbing surface where as in the case of random copolymers, only a certain fraction of monomers which are randomly distributed along the chain interact with the surface while the rest remain neutral. Previous studies ${ }^{9,10}$ gave reasonable descriptions of how the molecular architecture and sequence distribution affects the adsorption of copolymers. But the critical properties of the same remain still unknown. Our major aim in this investigation is to study this and to compare the results with that of homogeneous poly-

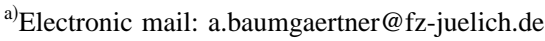

mer adsorbed on a homogeneous as well as on a chemically random surface. ${ }^{15}$

\section{MODEL AND SIMULATION TECHNIQUES}

We have used Monte Carlo simulation methods to investigate the adsorption characteristics of a self-avoiding random copolymer on a planar homogeneous surface. Our model consists of a cubic lattice of unit spacing, where the $z=0$ plane of the lattice represents the impenetrable, planar surface. The lattice has linear dimensions of $L_{x}=L$ and $L_{y}$ $=L$, with $L=300$ and $L_{z}=N$. We used periodic boundary conditions. The polymer chain is modeled as a self-avoiding walk of $N-1$ steps on the cubic lattice. Its chemical structure is designed as a random copolymer having two types of units $A$ and $B$. One of the monomer units $A$, has an attractive interaction of strength $\epsilon<0$ with the surface at $z=0$. Type $B$ units are not attractive, $\epsilon=0$. The initial configuration of the polymer is generated with one monomer attached to the surface by a random growth process taking into account of selfavoidance. Subsequent different chain configurations are generated using the standard kink-jump and crank-shaft algorithms and using periodic boundary conditions in the $x$ and $y$ directions. For a given configuration of the chain the number of attractive monomers $E$ in contact with the surface is calculated.

Statistical weights are assigned appropriately to different configurations using standard Metropolis rules. A move is accepted if: (1) the self-avoidance is maintained and (2) $\exp \left[\epsilon\left(n_{\text {old }}-n_{\text {new }}\right) / k_{B} T\right]>\eta$, where $1>\eta>0$ is a random number, $T$ is the temperature, and $k_{B}$ is the Boltzmann constant. For the rest of the paper we will measure the temperature in units of $\epsilon / k_{B}$ and hence $T \equiv k_{B} T / \epsilon$. Here, in this problem, we have two averages, one over all possible configurations of the polymer chain and the other over the quenched random sequence of adsorptive monomers of the polymer chain. We have performed quenched averages using 30 different random sequences.

There is always a finite probability for the chain to escape from the surface at all nonzero temperatures in this model. This tendency will be larger at high temperatures and also for short chains. We have suppressed this effect by imposing the condition that at least one of the beads of the 


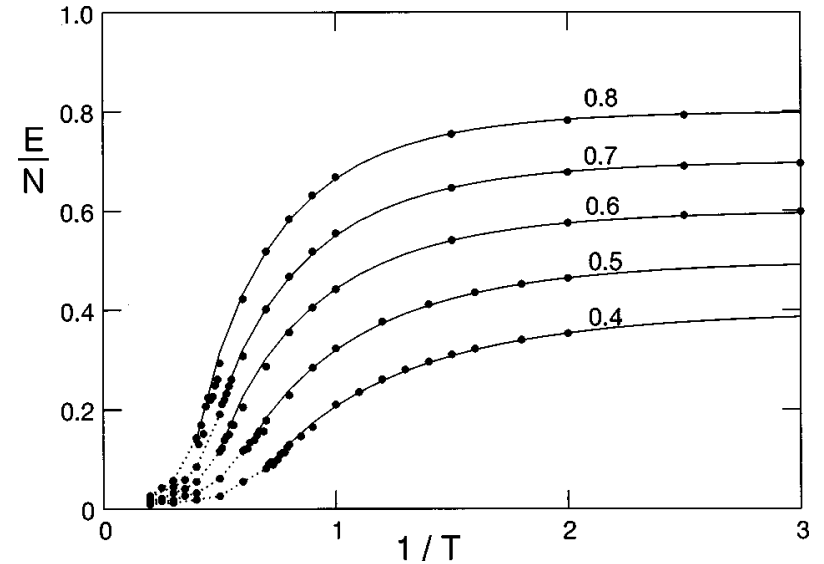

FIG. 1. Average energy of adsorption $E / N$ of random copolymers on the homogeneous surface versus temperature 1/T. The lines correspond to Eq. (22).

chain must be attached to the surface. This constraint, however, still allows the chain to diffuse in proximity to the surface and could be applied to the case of heterogeneous surfaces as well. ${ }^{15}$

In determining the averages, we have used $10^{6}$ Monte Carlo steps.

\section{RESULTS AND DISCUSSIONS}

We have estimated the average of the adsorption energy $E / N$ as a function of temperature $T$ for various chain lengths $N$ and for various fractions of attractive monomers $f$. The case where $f=1$ corresponds to adsorption of a homogeneous chain on a pure, planar surface. ${ }^{13}$ In the present investigation, we have studied polymer chains with lengths $N=75$, 100,150 and 200 and for values of $f=0.4,0.5,0.6,0.7,0.8$ and 1 . In the present study we used uncorrelated sequences of attractive and nonattractive monomers, where the probability of a monomer to be attractive is $f$.

The temperature dependence of $E / N$ for $N=100$ and for different values of $f$ is shown in Fig. 1. The other values of $N$ also show similar qualitative behavior. The adsorption energy per monomer $E / N$ shows a monotonous decrease with the increase in temperature. Near the critical temperature $T_{c}(N)$ the energy undergoes a significant change. It is clear from Fig. 1 that the adsorption energy do not only depends on $T$, but also on the fraction $f$. E/N increases with increasing $f$.

\section{A. The critical temperature}

It is evident from Fig. 1 that the value of $T_{c}(N)$ depends not only on the chain length $N$ but also on the fraction of adsorptive monomers and therefore we denote $T_{c}(N)$ as $T_{c}(N, f)$. The critical temperature $T_{c}(N, f)$ for each $N$ has been estimated for different values of $f$. This has been performed by taking the derivative of each curve $E / N$ vs $T$, as shown, for example, in Fig. 1 for the case $N=100$. The locations of the maxima of the derivatives have been identified with the transition temperatures $T_{c}(N, f)$. The results for $T_{c}(N, f)$ as a function of $N^{-\phi}$ are shown in Fig. 2 where $\phi$ is the crossover exponent. We used the value of $\phi=0.59$

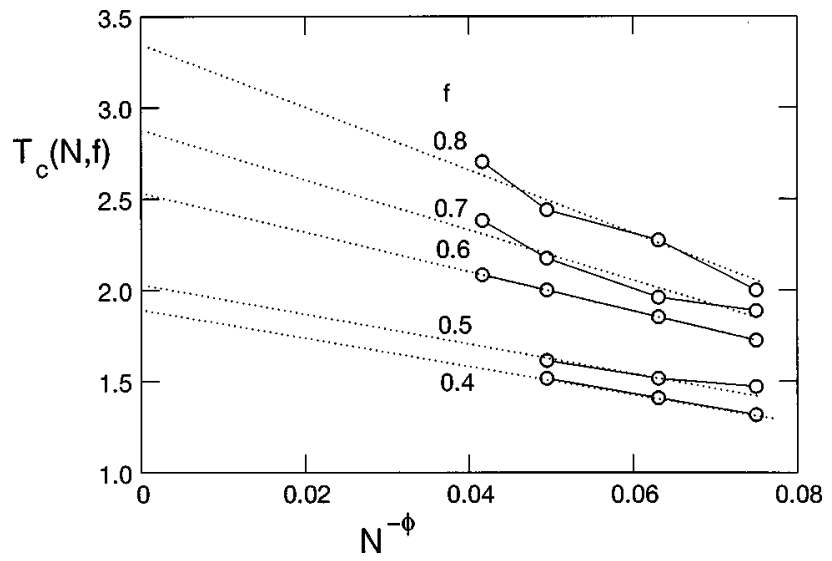

FIG. 2. Transition temperatures for different $f$ as a function of $N^{-\phi}$.

which is the same as that in the case of the adsorption of homopolymers. ${ }^{13}$ At this point we made the assumption that $\phi$ is the same for random copolymers independent of $f$. In fact, this will be justified in Sec. III B. By extrapolating each of the curves to very large $N$, we have estimated $T_{c}(f)$. The $T_{c}(f)$ values are then plotted against $f$ in Fig. 3. It is found that these data follow approximately a linear law $T_{c} \sim f$. However, in order to reconcile the critical temperature at $f=1, T_{c} \approx 3.3$, and the limiting case $f \rightarrow 0$, where $T_{c} \rightarrow 0$ should be expected, a better approximation is given by $T_{c}$ $=3.3 f^{0.7}$. The latter function is depicted in Fig. 3 by the solid line. It should be noted that a similar linear relationship between $T$ and $f$ has been predicted recently by Gutman and Chakraborty. ${ }^{12}$

\section{B. Critical properties}

Similarly as in the case for homopolymers ${ }^{13}$ we assume a scaling ansatz for the energy of the form

$$
E / N^{\phi}=h\left[\tau N^{\phi}, f\right],
$$

where $\tau=\left[T-T_{c}(f) / T_{c}(f)\right]$ is the temperature distance to the critical temperature. The scaling function has the asymptotic critical properties

$$
h(x) \propto\left\{\begin{array}{l}
x^{-1} f, \quad x \rightarrow \infty \\
\text { const. } \quad x \rightarrow 0 \\
|x|^{(1-\phi) / \phi} f, \quad x \rightarrow-\infty
\end{array},\right.
$$

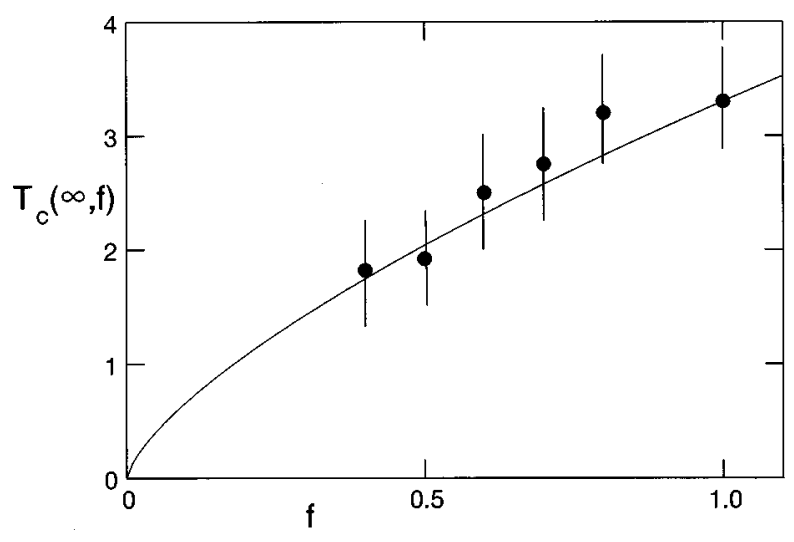

FIG. 3. $T_{c}(\infty, f)$ as a function of $f$. 


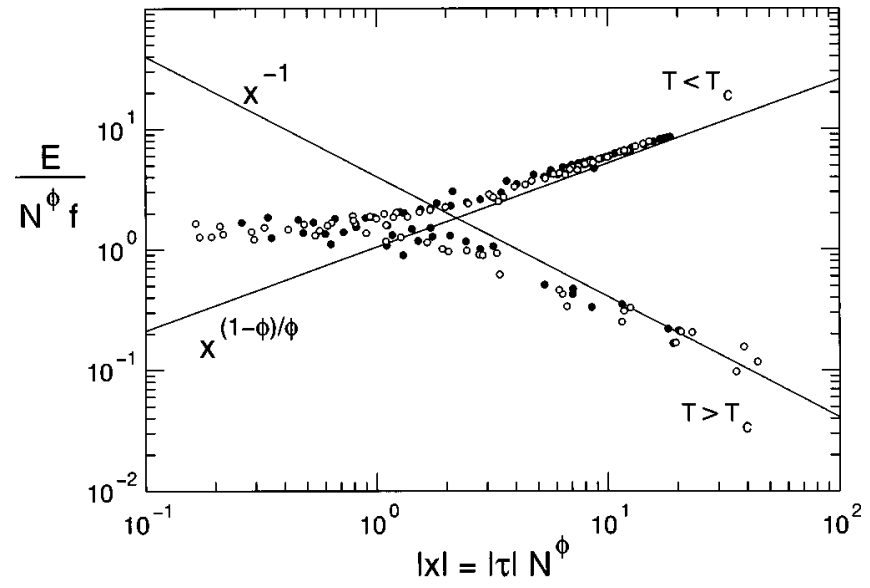

FIG. 4. The $\log -\log$ plot of $E / N^{\phi} f$ vs the scaling variable $|x|$ for $f=0.4$ and 0.8 denoted by open and full circles, respectively.

where $x=\tau N^{\phi}$. The corresponding asymptotic behavior $(|\tau| \ll 1, N \rightarrow \infty)$ is

$$
E / f N \propto\left\{\begin{array}{l}
{[|\tau| N]^{-1}, \quad T \geqslant T_{c}} \\
|\tau|^{(1-\phi) / \phi}, \quad T<T_{c} .
\end{array}\right.
$$

In Fig. 4 we present the corresponding scaling analysis of our energy data. For simplicity, only data for $f=0.4$ and $f$ $=0.8$ are presented. The straight lines drawn along each collapsed set of adsorption energy data in the figure give the expected asymptotic slopes according to the above scaling assumptions [Eq. (3)] which are in fair agreement with our Monte Carlo data. The crossover exponent has been fitted to $\phi=0.59$, similar to our previous studies on homogeneous polymer adsorption on homogeneous surfaces. ${ }^{13}$ The slightly smaller exponent of $\phi=0.54$ based on other calculations ${ }^{14}$ could not be confirmed. It is interesting to note that the crossover exponent is independent of $f$, which is quite different from the case of adsorption of homogeneous polymers on random surfaces. In the latter case the exponent seems to change continuously with the degree of randomness. ${ }^{15}$

Similar scaling analysis can be performed for the radius of gyration $R$ and its parallel and perpendicular components with respect to the surface $R_{\|}$and $R_{\perp}$, respectively. The appropriate scaling ansatz for these quantities are:

$$
R_{\perp}=N^{\nu} g_{\perp}\left[\tau N^{\phi}, f\right]
$$

and

$$
R_{\|}=N^{\nu} g_{\|}\left[\tau N^{\phi}, f\right] .
$$

The scaling functions $g_{\perp}(x, f)$ and $g_{\|}(x, f)$ are assumed to have the following aymptotic behavior:

$$
g_{\perp}(x, f) \propto\left\{\begin{array}{l}
\text { const., } \quad x \rightarrow \infty \\
\text { const., } \quad x \rightarrow 0 \\
|x|^{-\nu / \phi} \hat{g}_{\perp}(f), \quad x \rightarrow-\infty
\end{array}\right.
$$

and

$$
g_{\|}(x, f) \propto\left\{\begin{array}{l}
\text { const., } \quad x \rightarrow \infty \\
\text { const., } \quad x \rightarrow 0 \\
|x|^{\left(\nu_{2}-\nu\right) / \phi} \hat{g}_{\|}(f), \quad x \rightarrow-\infty .
\end{array}\right.
$$

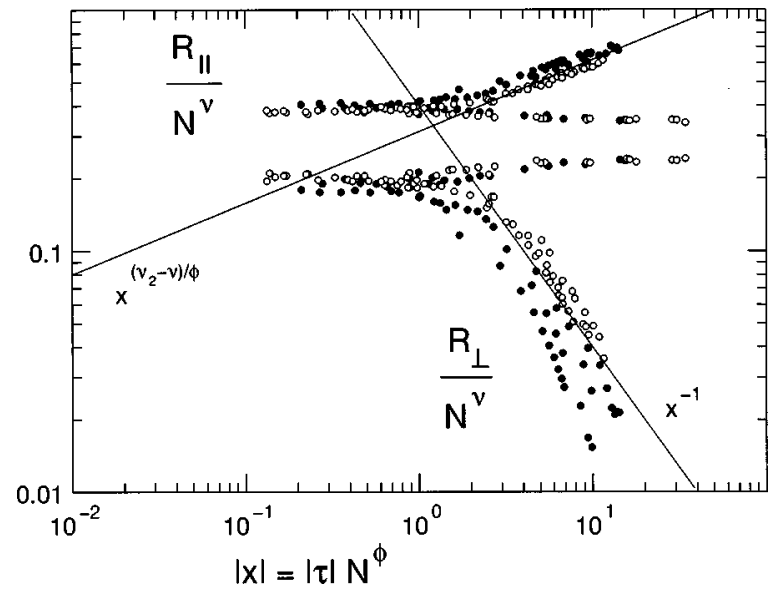

FIG. 5. The log-log plot of $R_{\perp} / N^{\nu}$ and $R_{\|} / N^{\nu}$ as a function of the scaling variable $x$ for $f=0.4$ (open symbols) and $f=0.8$ (full symbols).

The corresponding asymptotic behavior of the two components is:

$$
\begin{aligned}
& R_{\perp} \propto\left\{\begin{array}{l}
N^{\nu}, \quad T \geqslant T_{c} \\
|\tau|^{-\nu / \phi} \hat{g}_{\perp}(f), \quad T<T_{c} .
\end{array}\right. \\
& R_{\|} \propto\left\{\begin{array}{l}
N^{\nu}, \quad T \geqslant T_{c} \\
N^{\nu}|\tau|^{\left(\nu_{2}-\nu\right) / \phi} \hat{g}_{\|}(f), \quad T<T_{c},
\end{array}\right.
\end{aligned}
$$

where $\nu_{2}=3 / 4$ and $\nu \approx 0.59$ are the correlation length exponents for two and three dimensions, respectively. In Fig. 5 we present the scaling analysis of the Monte Carlo data of $R_{\perp}$ and $R_{\|}$, respectively. The upper and the lower wing of data correspond to the case $T<T_{c}$ for $R_{\|}$and $R_{\perp}$, respectively. The other data, almost independent of $|x|$, correspond to the case $T>T_{c}$. The straight lines drawn along each collapsed set of data in the figure give the expected asymptotic slopes according to the above scaling assumptions, Eqs. (8) and (9). The data are in good agreement for $R_{\|}$, but in poor agreement for $R_{\perp}$. The latter case might be attributed to large statistical errors in our data. Another possibility is that at low temperatures the component $R_{\perp}$ departs strongly from the power law, Eq. (8), which is observed in Fig. 5.

For the scaling functions, Eqs. (6) and (7), we have assumed that the variables $x$ and $f$ obey different functions where $\hat{g}_{\|}(f)$ and $\hat{g}_{\perp}(f)$ are unknown. However, a different scaling ansatz with a combined scaling variable is also conceivable, where

$$
R_{\perp}=N^{\nu} g_{\perp}\left[\tau N^{\phi} f^{\phi /(1-\phi)}\right]
$$

and

$$
R_{\|}=N^{\nu} g_{\|}\left[\tau N^{\phi} f^{\phi /(1-\phi)}\right] .
$$

The corresponding asymptotic behavior, analog to Eq. (6) and (7) where $x=\tau N^{\phi} f^{\phi /(1-\phi)}$ and $\hat{g}=1$, would give

$$
\begin{aligned}
& R_{\perp} \propto\left\{\begin{array}{l}
N^{\nu}, \quad T \geqslant T_{c} \\
|\tau|^{-\nu / \phi} f^{-\nu /(1-\phi)}, \quad T<T_{c},
\end{array}\right. \\
& R_{\|} \propto\left\{\begin{array}{l}
N^{\nu}, \quad T \geqslant T_{c} \\
N^{\nu}|\tau|^{\left(\nu_{2}-\nu\right) / \phi} f^{\left(\nu_{2}-\nu\right) /(1-\phi)}, \quad T<T_{c} .
\end{array}\right.
\end{aligned}
$$




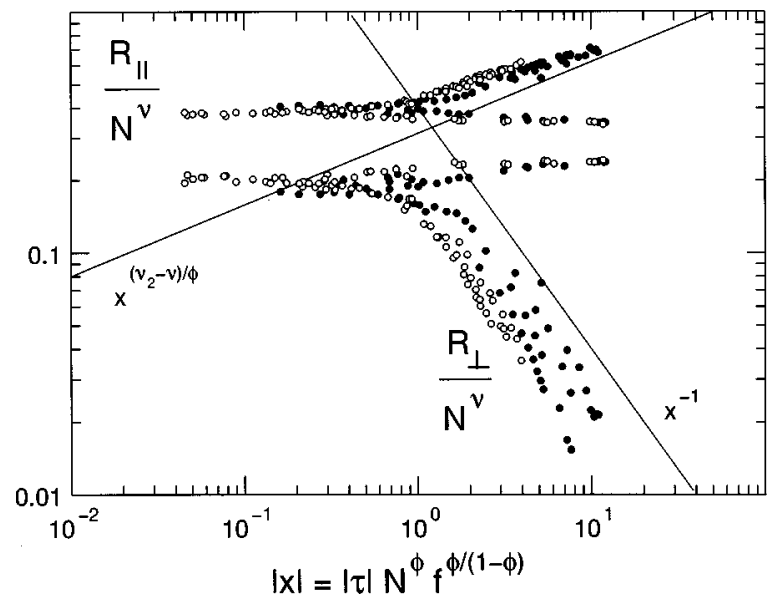

FIG. 6. The $\log -\log$ plot of $R_{\|} / N^{\nu}$ as a function of the scaling variable $x$.

The Monte Carlo data as a function of $x=\tau N^{\phi} f^{\phi /(1-\phi)}$ are given in Fig. 6. Although the collapse of the data has not significantly improved as compared to Fig. 5, the scaling assumptions Eqs. (10) and (11), seem to be the correct one, because the asymptotic power law, Eq. (12), is in agreement with predictions based on the proximal exponent and with our Monte Carlo data which is discussed below.

It should be noted that for the energy, a scaling similar to Eq. (10) could be assumed. Although this would give the correct low temperature behavior of Eq. (3), the high temperature behavior, would be wrong leading to $E$ $\sim 1 / f^{\phi /(1-\phi)}$, and implying that $E$ would increase with decreasing $f$.

\section{Low temperature properties}

The low temperature behavior of $R_{\perp}$ and in particular the power law of $f$ in Eq. (8) are related to the concentration profile of the monomers near the wall ${ }^{13,16}$

$$
p(z)=p_{0}\left(\frac{a}{z}\right)^{m} v\left(\frac{z}{R_{\perp}}\right),
$$

where $m$ is the proximal exponent, and $v(0)=1$ and $v(\infty)$ $=0$, and $a$ is the size of one monomer. The density profile is related to the fraction of adsorbed monomers $E / N$ [Eq. (3)] by

$$
\frac{E}{N}=a p_{0} / \int d z p(z) \sim\left(\frac{a}{R_{\perp}}\right)^{1-m},
$$

which can be reformulated to

$$
R_{\perp} \sim\left(\frac{E}{N}\right)^{-[1 /(1-m)]} .
$$

Since the free energy of adsorption per monomer is ${ }^{17}$

$$
\Delta F \approx-\frac{E}{N} \tau+\left(\frac{a}{R_{\perp}}\right)^{1 / \nu},
$$

the minimization of the free energy with respect to $R_{\perp}$ and using Eq. (16) provides the condition

$$
\tau=\left(\frac{a}{R_{\perp}}\right)^{1 / \nu+m-1} .
$$

Comparing this result with the asymptotic scaling form [Eq. (6)] for $T<T_{c}$, one gets the result

$$
\frac{1}{1-m}=\frac{\nu}{1-\phi}=1.44,
$$

where $\nu=\phi=0.59$. The Monte Carlo data of $R_{\perp}$ at low temperatures are approximately described by

$$
R_{\perp} \approx\left[f e^{-\left(T-b_{f}\right)}\right]^{-\nu /(1-\phi)},
$$

which is shown in Fig. 7. The constant $b_{f} \approx 1.6$ has been fitted to provide the extrapolated value of $R_{\perp} f^{-\nu /(1-\phi)}$ $\approx 0.1$ at $T=0$. The normalized data collapse to almost a single line, almost independent of $f$. The full line in Fig. 7 corresponds to Eq. (20). Although the main behavior of $R_{\perp}$ is well described by Eq. (20), there is still a weak dependence on $N$ leading to a scattering of the data. It should be noted that the above approximation for $R_{\perp}$ does not include the correct limit at $f=1$ and $T \rightarrow 0$, where $R_{\perp}=0$ must be expected. This effect is probably somehow buried in the constant $b_{f}$.

The Monte Carlo data of $R_{\|}$at low temperatures are approximately described by

$$
\frac{R_{\|}}{a N^{\nu_{2}}} \approx\left[f e^{-\left(T-c_{f}\right)}\right]^{\left(\nu_{2}-\nu\right) /(1-\phi)},
$$

where $a=0.327$ for strict self-avoiding walks on the square lattice and $\left(\nu_{2}-\nu\right) /(1-\phi)=0.39$. Similar to the case of $R_{\perp}$, the normalized data of $R_{\|}$collapse to almost a single line which is well approximated by Eq. (21) using the constant $c_{f} \approx 0.9$. This is shown in Fig. 8 .

At low temperatures all the energy curves in Fig. 1 attain their expected limiting values at $T \rightarrow 0$ of the ground states $E / N=f$. It has been suggested ${ }^{5,6}$ that the low temperature behavior of the energy is approximately given by $E / N$ $=f /\left[1+\exp \left(\epsilon / k_{B} T\right)\right]$. We found this approximation to be in a very poor agreement with our data. A different formula, valid for $T<T_{c}(N)$, is given by

$$
E / N=f \tanh \left[\frac{T_{c}(N)}{T^{*}(N)}\left(\frac{T^{*}(N)}{T}-1\right)^{(1-\phi) / \phi}\right],
$$

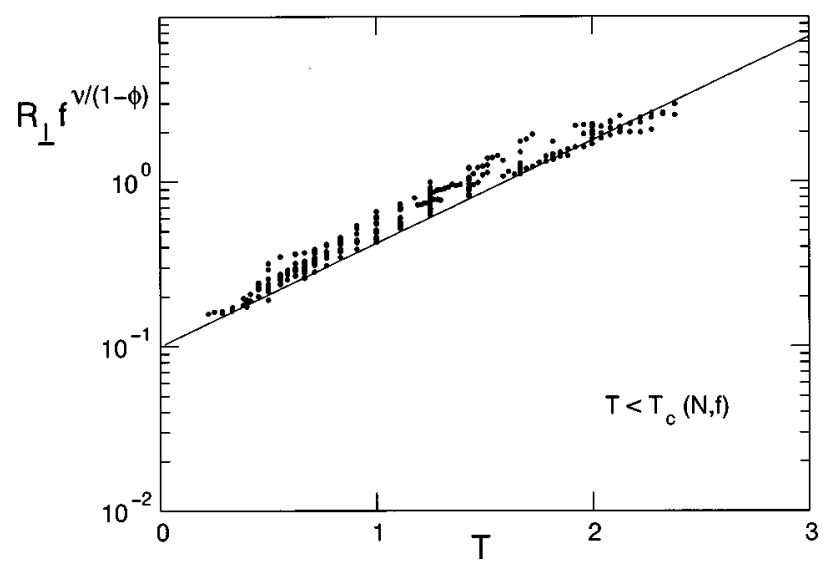

FIG. 7. The normalized perpendicular component $R_{\perp} f^{\nu /(1-\phi)}$ of the radius of gyration as a function of temperature. The line is given by Eq. (20). 


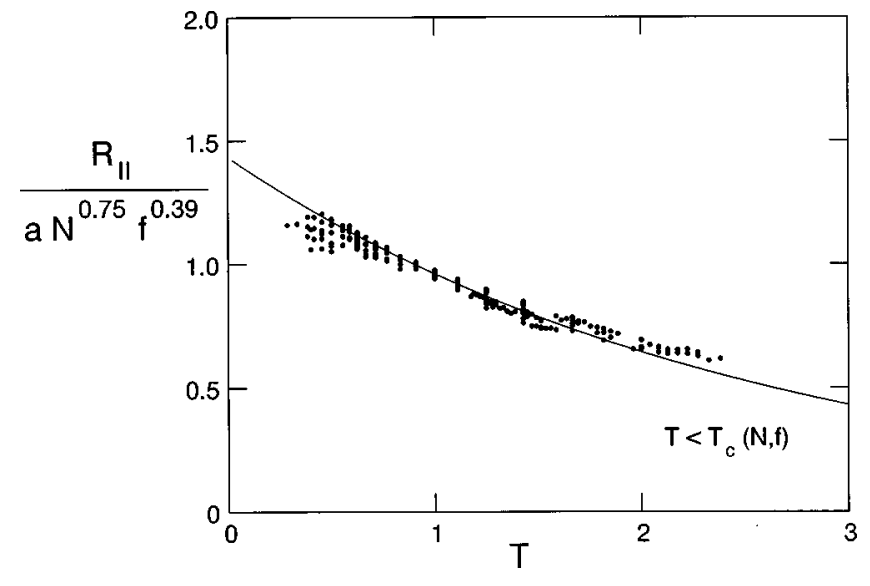

FIG. 8. The normalized parallel component $R_{\|}$of the radius of gyration as a function of temperature. The line is given by Eq. (21).

and is in excellent agreement with the data. This is depicted in Fig. 1 by the solid lines. The dotted lines in Fig. 1 simply connect the Monte Carlo data at $T>T_{c}$. The "effective critical temperature" $T^{*}(N)$ is related to the critical temperature by $T_{c}(N) / T^{*}(N) \approx 0.8$ for approximately all $N$. Therefore, Eq. (1) describes not only the data for $N=100$, as shown in Fig. 1, but also the other data for $N=75,150$, and 200 .

\section{SUMMARY AND CONCLUSIONS}

Here we report our results of Monte Carlo simulations of a random copolymer adsorbed on a homogeneous surface. It is found that the critical crossover exponent $\phi$ is unaltered with respect to the case of homogeneous polymers. However, the scaling behavior is changed by the fraction $f$ of adsorptive monomers of the chain as compared to the homogeneous case. In particular, we suggest some explicit expressions for energy and radius of gyration at low temperatures.

Finally it should be noted that it would be of interest to perform more detailed comparisons with the mean-field theory of Gutman and Chakraborty ${ }^{12}$ in the future.

\section{ACKNOWLEDGMENT}

Financial support to one of the authors (K.S.) by the Forschungszentrum is gratefully acknowledged.

${ }^{1}$ T. Garel, D. A. Huse, S. Leibler, and H. Orland, Europhys. Lett. 8, 9 (1989).

${ }^{2}$ T. Garel, H. Orland, and E. I. Shaknovich, J. Chem. Phys. 93, 2043 (1990).

${ }^{3}$ C. Yeung, A. C. Balazs, and D. Jasnow, Macromolecules 25, 1357 (1992).

${ }^{4}$ W. Li, C. Yeung, D. Jasnow, and A. C. Balazs, Macromolecules 25, 3685 (1992).

${ }^{5}$ J. F. Joanny, J. Phys. II (France) 4, 1281 (1984).

${ }^{6}$ J. C. Shaffer, Macromolecules 28, 7447 (1995).

${ }^{7}$ S. Srebnik, A. K. Chakraborty, and E. I. Shaknovich, Phys. Rev. Lett. 77, 3157 (1996)

${ }^{8}$ A. K. Chakraborty and D. Bratko, J. Chem. Phys. 108, 1676 (1998).

${ }^{9}$ A. C. Balazs, M. Gempe, and C. W. Lantman, Macromolecules 24, 168 (1991).

${ }^{10}$ E. A. Zheligovskaya, P. G. Khalatur, and A. R. Khokhlov, J. Chem. Phys. 106, 8588 (1997)

${ }^{11}$ L. Gutman and A. K. Chakraborty, J. Chem. Phys. 101, 10074 (1994).

${ }^{12}$ L. Gutman and A. K. Chakraborty, J. Chem. Phys. 103, 10733 (1995).

${ }^{13}$ E. Eisenriegler, K. Kremer, and K. Binder, J. Chem. Phys. 77, 6296 (1982).

${ }^{14}$ D. Zhao, T. Lookman, and K. De'Bell, Phys. Rev. A 42, 4591 (1990).

${ }^{15}$ K. Sumithra and A. Baumgaertner, J. Chem. Phys. 109, 1540 (1998).

${ }^{16}$ P. G. de Gennes and P. Pincus, J. Phys. Lett. (France) 44, L241 (1983).

${ }^{17}$ P. G. de Gennes, Scaling Concepts on Polymer Physics (Cornell University Press, Ithaca, NY, 1979). 